\title{
Analytical Multiloop Control for Multivariable Systems with Time Delays
}

\author{
Zhiguo Wang $(\mathbb{D}$ and Peng Wei \\ Key Laboratory of Advanced Process Control for Light Industry (Ministry of Education), Institute of Automation, \\ Jiangnan University, Wuxi 214122, China
}

Correspondence should be addressed to Zhiguo Wang; zhiguowang@jiangnan.edu.cn

Received 6 August 2020; Revised 2 September 2020; Accepted 8 September 2020; Published 14 September 2020

Academic Editor: Shuping He

Copyright $(92020$ Zhiguo Wang and Peng Wei. This is an open access article distributed under the Creative Commons Attribution License, which permits unrestricted use, distribution, and reproduction in any medium, provided the original work is properly cited.

\begin{abstract}
In this paper, a new design method with performance improvements of multiloop controllers for multivariable systems is proposed. Precise expression is developed to show the relationship between the dynamic- and steady-state characteristics of the multiloop control system and its parameters. First, an equivalent transfer function (ETF) is introduced to decompose the multivariable system, based on which the multiloop controller parameters are calculated. According to the ETF matrix property, an analytical expression for the PI controller for multivariable systems is derived in terms of substituting the ETF matrix for the inverse open-loop transfer function. In the proposed controller design method, no approximation of the inverse of the process model is needed, implying that this method can be applied to some multivariable systems with high dimensions. The simulation results obtained from several examples demonstrate the effectiveness of the proposed method.
\end{abstract}

\section{Introduction}

In the actual industrial process, especially in multivariable systems such as petroleum, chemical, and biological, the interconnections introduced by the process configuration and control architecture are very common [1]. Due to the existence of these interconnection characteristics, a disturbance or a small change of process variable in one or more loops would be propagated to other loops, and in turn, feedback to the source loop to some extent, thereby critically affecting the controller performance and stability of the overall system. At the same time, this interconnection phenomenon makes the design process of multivariable controllers very complicated.

Compared to the single loop system, the multiloop control system's design issues become very complicated as a result of these interconnections, which make it an open topic for many years. There are many reports on this field in the existing literature. From the perspective of controller structure design, these methods can classify into three categories, namely, decoupling plus single loop controller structure, centralized control structure, and multiloop control structure. The classic multiloop PID controller has been widely used in processes with moderate interaction due to some advantages, such as simple control structure, less adjustable parameters, and easy to understand features. For example, a multiloop controller design method is given by Huang et al. [2]. In this method, the multivariable target systems are decomposed into several single but equivalent loops for design. By doing this, prior information of controller dynamics in other loops is no longer needed in each loop, resulting in an interesting method designing controller directly and independently. Shen et al. [3] designed selected loop controllers independently based on equivalent transfer functions. $\mathrm{Vu}$ and Lee [4] propose an independent design method for multivariable processes, and firstly the multiloop system is decomposed into a set of single loops using effective open-loop transfer function (EOTF); then, the individual controller of every single loop is designed based on the corresponding EOTF model. Estévez-Sánchez et al. [5] proposed a design method of a multiloop PI controller with the linear quadratic regulator (LQR) methodology. 
For the same target, meanwhile, the multiple time delays commonly occur in multivariable processes of high complexity. Particular examples can be found, such as pilot plant distillation columns and high purity distillation columns. The essence of the reason for time delay is the complex interactions between the numerous different pairs of input and output variables. Therefore, approximating the actual model by a reduced-order form by using some model reduction techniques is necessary, for example, the first-order plus dead time (FOPDT) and second-order plus dead time (SOPDT). On the contrary, for systems with time delays, the internal model control (IMC) method is a very practical approach for the design of IMC-PID controllers. Several results have been discussed and reported with some interesting results, extending the IMC-PID of the single-input and singleoutput (SISO) case to the multivariable case [6]. To overcome the above problems or drawbacks, an intuitive solution is designed multivariable controllers that are fully crosscoupled. To implement this kind of controllers, however, it is not an easy job since all the computation of control input is coupled, and the designers are required to compute the inverse of the transfer function matrix. For the system of high dimensions, the inverse of the transfer function can be quite complex and hard to be determined. Furthermore, based on the approach of direct synthesis, $\mathrm{Vu}$ and Lee [7] proposed a method of analytical solution for the design of the PI controller in a multiloop system. As the complication to obtain the inversion of the transfer function, the method has the limitation of only suitable for low-dimensional multivariable systems.

Motivated by the above, a novel decoupling method is proposed for multivariable control systems in this paper. By taking the essential effects of the interactions into account, we use the concept of the equivalent transfer function (ETF) to decompose the multivariable system under consideration into multiple univariate loops equivalently. After that, the properties of the ETF matrix and the target closed-loop diagonal transfer function that was specified to the nonminimum phase zeros and inherent time delays are used to derivate the controller parameters. The relationship between proposed controller parameters and the open-loop transfer function is obtained without the prior acknowledge of the specific ETF model. We have established some equations directly to deduce the parameters of the multiloop PI controller. Finally, the proposed approach is applied to different industrial objects to verify its effectiveness.

\section{Preliminaries}

In the existing literatures, the idea that decomposes a system of the multiloop controller into several signal loops equivalently is the main approach to design controller for the multiloop system. By doing this, the next step is to design the controller for the transfer function obtained with open-loop feature. To give more details, for ETF loop $i$, its open-loop function is achieved as the transfer function of $u_{i}$ with $y_{i}$, where only the loop $i$ is open and all the other loops are closed. We show this idea by Figure 1, where the function of $u_{i}$ to other outputs is indicated by the $y_{i}$, while the effect of $y_{i}$ by all the other control loops with closed feature is indicated by $\bar{y}_{i}$. The $\mathbf{u}^{i}$ and $\mathbf{y}^{i}$ represent $r, u$, and $y$ with the corresponding $i$ th elements, $r_{i}, u_{i}, y_{i}$, and $c_{i}(s)$, removed, respectively. Once the ETF is obtained, model reduction was used to approximate the ETF to FOPDT or SOPDT model. Therefore, some classic and well-understood PID parameter tuning methods for common univariate system can be used in the design of each individual PID controller obtained here.

The IMC-PID controller tuning method introduced by Lee et al. [8] is used to calculate the parameter of controller. The reduced ETF, $\widehat{g}_{i i}$, is decomposed to $\widehat{g}_{i i}=\widehat{g}_{m, i i} \widehat{g}_{p, i i}$, where $\hat{g}_{m, i i}$ and $\hat{g}_{p, i i}$ are the minimum phase portion and nonminimum phase portion, respectively. Through simple derivation, we can get the desired closed-loop response under the action of the ideal feedback controller, which is given by

$$
g_{c i}=\frac{q_{i}}{\left(1-\widehat{g}_{i i}\right)}=\frac{\widehat{g}_{m, i i}^{-1}(s)}{\left(\lambda_{i} s+1\right)-\widehat{g}_{p, i i}(s)},
$$

where $q_{i}$ is the IMC controller and is designed by

$$
q_{i}=\widehat{g}_{i i}^{-1} f_{i} \text {. }
$$

The IMC filter, $f(s)$, can be rewritten in the form of $f_{i}(s)=1 /\left(\lambda_{i} s+1\right)^{m_{i}}$, where the adjustable filtering parameter $\lambda_{i}$ balances the trade-off between performance and robustness. Besides, to ensure the controller is practical and suitable, the order parameter $m_{i}$ is selected as a positive integer.

To use the above controller as the standard PID form, we need to approximate the feedback controller $g_{c i}$ by the PI controller forms of equivalent property. To this end, expanding $g_{c i}$ by the Maclaurin series in $s$ yields the following equation:

$$
g_{c i} \equiv \frac{f_{i}(s)}{s}=\frac{1}{s}\left[f_{i}(0)+f_{i}^{\prime}(0) s+\frac{f_{i}^{\prime \prime}(0)}{2 !} s^{2}+\cdots\right] .
$$

The controller stated by equation (3) can be interpreted as the traditional PID controller by using the first two terms given by

$$
g_{c i}(s)=k_{c i}\left(1+\frac{1}{\tau_{I i} s}\right)
$$

where

$$
\begin{aligned}
k_{c i} & =f_{i}^{\prime}(0), \\
\tau_{I i} & =\frac{f_{i}^{\prime}(0)}{f_{i}(0)} .
\end{aligned}
$$

Through the above descriptions, the concept of the ETF is introduced to solve the controllers design problem. In the following section, we will utilize the property of the ETF matrix to find the important relationship between the parameters of the controller and that of the considered model. 


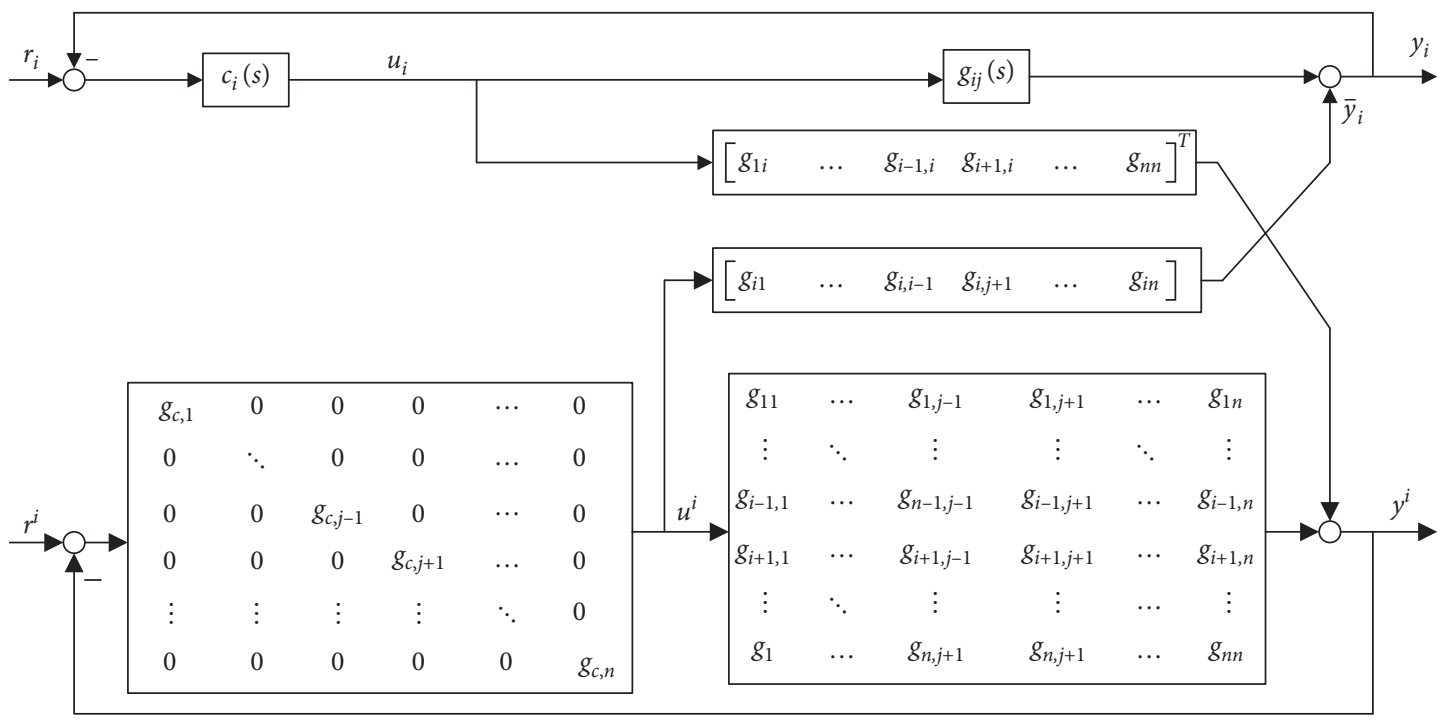

Figure 1: Block diagram for the concept of the ETF.

\section{Design of Multiloop Controller}

3.1. Controller Parameter Design. For a multivariable system with closed-loop controllers, we can write the forward transfer function to be of the form [9]

$$
G(s) G_{c}(s) \approx G(s) G^{-1}(s)=\frac{I}{s} .
$$

In the above formula, $G(s)$ is the process transfer function matrix and $G_{c}(s)$ represents diagonal controller matrix of multivariable system. The detailed structure of these two matrices is as follows:

$$
\begin{gathered}
G(s)=\left[\begin{array}{ccc}
g_{11}(s) & \cdots & g_{1 n}(s) \\
\vdots & \ddots & \vdots \\
g_{n 2}(s) & \cdots & g_{n n}(s)
\end{array}\right], \\
G_{c}(s)=\left[\begin{array}{ccc}
g_{c, 11}(s) & \cdots & 0 \\
\vdots & \ddots & \vdots \\
0 & \cdots & g_{c, n n}(s)
\end{array}\right] .
\end{gathered}
$$

Due to the right half plane zeros and time delays in multivariable systems, which make an ideal controller that is equal to the inverse of open-loop transfer function matrix unrealizable, the closed-loop transfer function matrix of the IMC system [10] is given to

$$
G(s) G_{c}(s)=H(s)=\operatorname{diag}\left\{h_{i}\right\}, \quad i=1,2, \ldots, n,
$$

where

$$
h_{i}=e^{-\left(\tau(G)-\tau_{i}\right) s} \prod_{z \in Z_{|G|}^{+}}\left(\frac{z-s}{z+s}\right)^{\eta_{z}(|G|)-\eta_{i}(z)},
$$

$\left(\tau(G)-\tau_{i}\right)$ is the largest time delay of $i$ th row element of $G(s)$ and $\eta_{z}(|G|)-\eta_{i}(z)$ is on its, $h_{i}$, the nonminimum phase zeros. In order to ensure system stability and realization, we should restrict on the following conditions:

$$
\begin{aligned}
\tau\left(h_{i}\right) & \geq \tau(|G|)-\tau_{i}, \\
\eta_{z}\left(h_{i}\right) & \geq \eta_{z}(|G|)-\eta_{z}(z) .
\end{aligned}
$$

According to the properties of the ETF, we have

$$
G^{-1}(s)=\left[\begin{array}{ccc}
g_{11}(s) & \cdots & g_{1 n}(s) \\
\vdots & \ddots & \vdots \\
g_{n 1}(s) & \cdots & g_{n n}(s)
\end{array}\right]^{-1}=\left[\begin{array}{ccc}
\frac{1}{\widehat{g}_{m, 11}} & \cdots & \frac{1}{\hat{g}_{m, 1 n}} \\
\vdots & \ddots & \vdots \\
\frac{1}{\widehat{g}_{m, n 1}} & \cdots & \frac{1}{\widehat{g}_{m, n n}}
\end{array}\right]^{T}
$$

Comparing equations (1) and (9) and from literatures $[11,12]$, we can obtain

$$
\left[\begin{array}{ccc}
g_{11}(s) & \cdots & g_{1 n}(s) \\
\vdots & \ddots & \vdots \\
g_{n 1}(s) & \cdots & g_{n n}(s)
\end{array}\right]\left[\begin{array}{ccc}
\frac{1}{\hat{g}_{m, 11}} & \cdots & \frac{1}{\hat{g}_{m, 1 n}} \\
\vdots & \ddots & \vdots \\
\frac{1}{\hat{g}_{m, n 1}} & \cdots & \frac{1}{\hat{g}_{m, n n}}
\end{array}\right]=\left[\begin{array}{ccc}
h_{1}(s) & \cdots & 0 \\
\vdots & \ddots & \vdots \\
0 & \cdots & h_{n}(s)
\end{array}\right] .
$$

Then,

$$
\left[\begin{array}{ccc}
\frac{1}{\widehat{g}_{m, 11}} & \cdots & \frac{1}{\widehat{g}_{m, 1 n}} \\
\vdots & \ddots & \vdots \\
\frac{1}{\hat{g}_{m, n 1}} & \cdots & \frac{1}{\hat{g}_{m, n n}}
\end{array}\right]=\left[\begin{array}{ccc}
g_{11}(s) & \cdots & g_{1 n}(s) \\
\vdots & \ddots & \vdots \\
g_{n 1}(s) & \cdots & g_{n n}(s)
\end{array}\right]^{-1}\left[\begin{array}{ccc}
h_{1}(s) & \cdots & 0 \\
\vdots & \ddots & \vdots \\
0 & \cdots & h_{n}(s)
\end{array}\right] \text {. }
$$

In literatures $[4,7]$, for a weak coupling between each loop, a multiloop controller will give satisfied performance. By means of equation (14), we summarize it as follows: 


$$
\begin{array}{r}
\frac{1}{\hat{g}_{m, i i}}=\left\{G^{-1}(s) H\right\}_{i i}, \\
\hat{g}_{p, i i}=h_{i} .
\end{array}
$$

From equation (1), we can obtain

$$
g_{c i}=\frac{\left\{G^{-1}(s) H\right\}_{i i}}{\left(\lambda_{i} s+1\right)-h_{i}}=\frac{\left\{G^{-1}(s)\right\}_{i i} h_{i}}{\left(\lambda_{i} s+1\right)-h_{i}} .
$$

Similar to equations (5) and (6), the PI controller parameters are calculated as

$$
\begin{gathered}
k_{I, i i}=\left.\frac{\operatorname{adj} K_{i i}}{|K|} \frac{s h_{i i}}{1-h_{i i}}\right|_{s=0}, \\
k_{C, i i}=\left.\left(\frac{\operatorname{adj} G_{i i}}{|G|} \frac{s h_{i}}{1-h_{i}}\right)^{\prime}\right|_{s=0}=\left.\frac{\operatorname{adj} K_{i i}}{|K|}\left(\frac{s h_{i}}{1-h_{i}}\right)^{\prime}\right|_{s=0} \\
-\left.\frac{1}{|K|^{2}}\left(\sum_{p=1}^{n}\left(\sum_{q=1}^{n}\left(\operatorname{adj} K_{i q}\right) g_{q p}^{\prime}\right) \operatorname{adj} K_{p i}\right) \frac{s h_{i}}{1-h_{i}}\right|_{s=0},
\end{gathered}
$$

where $g_{q p}{ }^{\prime}$ represents the first derivative of $g_{q p}$ and

$$
|K|=\left|\begin{array}{ccc}
k_{11} & \cdots & k_{1 n} \\
\vdots & \ddots & \vdots \\
k_{n 1} & \cdots & k_{n n}
\end{array}\right|
$$

For the convenience of presentation, we simplified $\operatorname{adj} K_{j i}$, adj $K_{j p}$, and adj $K_{p i}$ into the same formula using subscripts $v$ and $w$, which are defined as

$\operatorname{adj} K_{v w}=(-1)^{v+w}\left|\begin{array}{cccccc}k_{11} & \cdots & k_{1, v-1} & k_{v+1} & \cdots & k_{1, n} \\ \vdots & \ddots & \vdots & \vdots & \ddots & \vdots \\ k_{w-1,1} & \cdots & k_{w-1, v-1} & k_{w-1, v+1} & \cdots & k_{w-1, n} \\ k_{w+1,1} & \cdots & k_{w+1, v-1} & k_{w+1, v+1} & \cdots & k_{w+1, n} \\ \vdots & \ddots & \vdots & \vdots & \ddots & \vdots \\ k_{n, 1} & \cdots & k_{n, v-1} & k_{n, v+1} & \cdots & k_{n, n}\end{array}\right|$,

where $k_{v w}$ is the steady gain.

Based on the analysis conventional methods to calculate the PI parameters, which decomposed multivariable systems to a set of multiloop control systems, then utilized singleinput and single-output design technique, we establish some equations to deduce and solve the parameters of PI directly and omit the intermediate step, i.e., how to calculate ETF parameters. This section derivates a direct relationship between the parameters of the multiloop controller and that of the open-loop transfer function matrix.

3.2. Performance of the Control System. To demonstrate the robust stability and also show its distinctive features, a popular and commonly used method of analysing robust stability is used in the presence of other controller design approaches. We use the output multiplication uncertainties to measure the robustness of the target system instead of input uncertainties, due to its advantage of less restrictive [4]. For the following system specified with an output uncertainty by

$$
\Delta G_{0}(s)=G(s)\left[I+\Delta_{0}(s)\right],
$$

where $\Delta_{0}(s)$ denotes the uncertainties occurring at multiplicative outputs, if the following relationship is found, we can conclude that the closed-loop system is stable:

$$
\gamma<\frac{1}{\bar{\sigma}}\left[\left(I+G(j w) G_{c}(j w)\right)^{-1} G(j w) G_{c}(j w)\right],
$$

where $\gamma$ is used to indicate the robust stability and $\bar{\sigma}$ represents maximum singular value. The larger the value of $\gamma$ is, the better the robust stability is.

The error integration criterion is a performance index, which is computed by the integral of the deviation between the actual output and the expected output. The integral absolute error (IAE) index and integrated time absolute error (ITAE) index are widely used because of their good practicability and selectivity. The IAE is introduced as follows :

$$
\operatorname{IAE}=\int_{0}^{\infty}|e(t)| \mathrm{d} t
$$

where

$$
e(t)=r(t)-y(t)
$$

To analyse the underlying performance, the IAE is first employed to evaluate the control performance of the target closed-loop systems. After that, the ITAE criterion comes in to give a new indicator showing closed-loop performance from the initial point to time infinity:

$$
\operatorname{ITAE}=\int_{0}^{\infty} t|e(t)| \mathrm{d} t
$$

The system is optimal, which means the IAE and the ITAE get smaller than the relative methods.

\section{Examples and Results}

In order to illustrate the control performance and robustness of the controller achieved by the proposed method, three typical industrial examples are used for testing. To be specific, the purpose of Example 1 and Example 2 is to show that the proposed method provides better performance than other existing methods for nominal systems. The main target of Example 3 is to demonstrate the performance of the proposed method to the systems of high dimensions.

4.1. Example 1. Consider the VL process [11], in which transfer function is given as

$$
\left[\begin{array}{cc}
\frac{-2.2 e^{(-s)}}{7 s+1} & \frac{1.3 e^{(-0.3 s)}}{7 s+1} \\
\frac{-2.8 e^{(-1.8 s)}}{9.5 s+1} & \frac{4.3 e^{(-0.35 s)}}{9.2 s+1}
\end{array}\right]
$$


We compare the multiloop tuning results obtained by the proposed method with those presented by Lee et al. [12] and Chen and Seborg [13]. For a fair comparison, we adjust the $\lambda_{i}$ used in the proposed method to be the same value of $\gamma$ or larger than that of others. The control parameters used in the process are shown in Table 1.

Figure 2 shows the closed-loop response performance of the method developed in this paper and the algorithms introduced by $[12,13]$, where the sequential step changes are set at $t=0$ and $t=150$ to the first loop and second loop. From Figure 2, it is clear to see that the PI controller designed by the method proposed in this paper has a faster response than the other two methods and has well-balanced responses.

To further investigate the robustness of the proposed method, a perturbation uncertainty of $+50 \%$ in process gain is inserted to the simulation study. As shown in Table 2, the $\mathrm{IAE}_{s}$ and $\mathrm{ITAE}_{s}$ values are smaller than those obtained by traditional methods. This verifies that the proposed method provides with better robust performance, where $\mathrm{IAE}_{s}$ and $\mathrm{ITAE}_{s}$ are the sum values of IAE and ITAE for each loop.

4.2. Example 2. Consider a $3 \times 3$ multivariable system:

$$
\left[\begin{array}{ccc}
\frac{0.66 e^{(-2.6 s)}}{6.7 s+1} & \frac{-0.61 e^{(-3.5 s)}}{8.64 s+1} & \frac{-0.0049 e^{(-s)}}{9.06 s+1} \\
\frac{1.11 e^{(-6.5 s)}}{3.25 s+1} & \frac{-2.36 e^{(-3 s)}}{5 s+1} & \frac{-0.012 e^{(-1.2 s)}}{7.09 s+1} \\
\frac{-33.68 e^{(-9.2 s)}}{8.15 s+1} & \frac{46.2 e^{(-9.4 s)}}{10.9 s+1} & \frac{0.87 e^{(-s)}(11.61 s+1)}{(3.89 s+1)(18.8 s+1)}
\end{array}\right]
$$

According to equations (17) and (18), the controller parameters are calculated in Table 3. Multiloop tuning results presented by literatures $[14,15]$ are employed to compare with the proposed methods.

Figure 3 gives the closed-loop responses of the proposed method and the compared methods, where the sequential step changes are made at $t=0, t=300$, and $t=600$ to the first, second, and third loop. From Figure 3, we can see that the fluctuation degree of the output signal corresponding to the proposed method is obviously smaller than that of other methods, and the steady state can be reached quickly.

Similar to the previous method, we also insert a perturbation uncertainty of $+50 \%$ in process gain to test the robust performance. The performance indices results are shown in Table 4; the $\mathrm{IAE}_{s}$ and $\mathrm{ITAE}_{s}$ values show that the proposed method in this paper has better robust performance.

4.3. Example 3. To further illustrate the effectiveness of the proposed method in high-dimensional systems, a system of four dimensions is introduced to verify the control performance and robustness. The corresponding transfer function of A2 system [16] is stated as

$$
\left[\begin{array}{cccc}
\frac{4.09 e^{(-1.3 s)}}{(33 s+1)(8.3 s+1)} & \frac{-6.36 e^{(-0.2 s)}}{(31.6 s+1)(20 s+1)} & \frac{-0.25 e^{(-0.4 s)}}{21 s+1} & \frac{-0.49 e^{(-5 s)}}{(22 s+1)^{2}} \\
\frac{-4.17 e^{(-4 s)}}{45 s+1} & \frac{6.93 e^{(-1.01 s)}}{44.6 s+1} & \frac{-0.05 e^{(-5 s)}}{(34.5 s+1)^{2}} & \frac{1.53 e^{(-2.8 s)}}{48 s+1} \\
\frac{-1.73 e^{(-17 s)}}{(13 s+1)^{2}} & \frac{5.11 e^{(-11 s)}}{(13.3 s+1)^{2}} & \frac{4.61 e^{(-1.02 s)}}{18.5 s+1} & \frac{-5.48 e^{(-0.5 s)}}{15 s+1} \\
\frac{-11.18 e^{(-2.6 s)}}{(43 s+1)(6.5 s+1)} & \frac{14.04 e^{(-0.02 s)}}{(45 s+1)(10 s+1)} & \frac{-0.1 e^{(-0.05 s)}}{(31.6 s+1)(5 s+1)} & \frac{4.49 e^{(-0.6 s)}}{(48 s+1)(6.3 s+1)}
\end{array}\right] .
$$

Using equations (27) and (28), the controller parameters are obtained in Table 5. Since Shen et al. [3] and He et al. [16] designed the controller by utilizing the simple IMC method [17] and gain phase margin, the parameter $\lambda_{i}$ is not adjusted in this simulation.
The curves in Figure 4 show the closed-loop time response of the proposed method proposed in this paper as well as the other two methods reported in literature $[3,16]$. It can be seen that, except for the lager overshoot of signal $y_{1}$, other signals have good dynamic- and steady-state 
TABle 1: Controller parameters.

\begin{tabular}{|c|c|c|c|c|c|}
\hline Methods & Loop & $k_{C, i j}$ & $k_{I, i j}$ & $\lambda_{i}$ & $\gamma$ \\
\hline \multirow{2}{*}{ Proposed } & 1 & -1.7730 & -0.2710 & 1.72 & \multirow{2}{*}{0.59} \\
\hline & 2 & 2.9550 & 0.3436 & 0.75 & \\
\hline \multirow{2}{*}{ Lee } & 1 & -1.3100 & 0.5800 & - & \multirow{2}{*}{0.53} \\
\hline & 2 & 3.9700 & 1.6400 & - & \\
\hline \multirow{2}{*}{ Chen } & 1 & 1.2100 & 0.2600 & - & \multirow{2}{*}{0.50} \\
\hline & 2 & 3.7400 & 3.4000 & - & \\
\hline
\end{tabular}
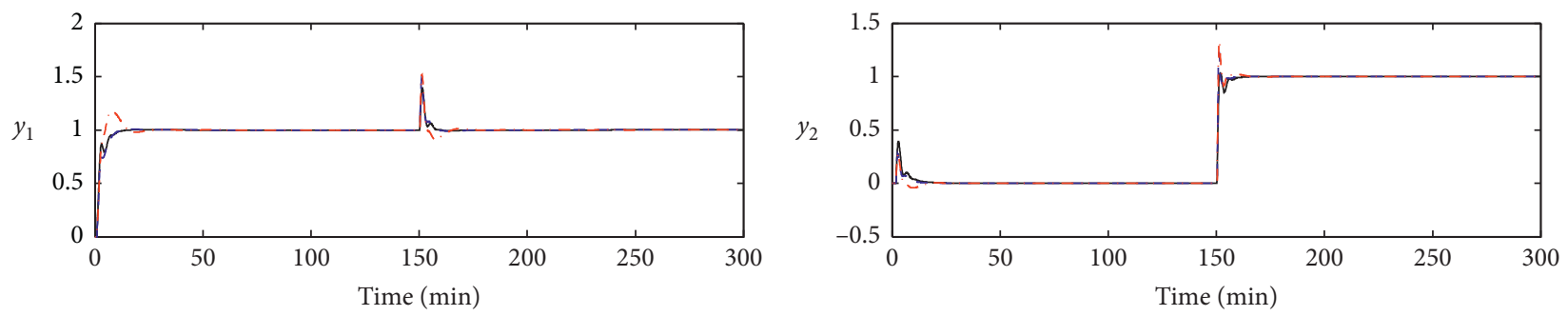

- Proposed

- - - Lee et al.

.... Chen et al.

$$
\begin{array}{ll}
\text { _ } & \text { Proposed } \\
\text { _- } & \text { Lee et al. } \\
\ldots & \text { Chen et al. }
\end{array}
$$

(a)

(b)

\begin{tabular}{|c|c|c|c|c|}
\hline \multirow{2}{*}{ Methods } & \multicolumn{2}{|c|}{$\mathrm{IAE}_{s}$} & \multicolumn{2}{|c|}{$\mathrm{ITAE}_{s}$} \\
\hline & Nominal & $+50 \%$ & Nominal & $+50 \%$ \\
\hline Proposed & 6.391 & 6.007 & 22.675 & 19.24 \\
\hline Lee & 7.102 & 6.899 & 32.369 & 23.99 \\
\hline Chen & 7.370 & 7.506 & 27.066 & 25.23 \\
\hline
\end{tabular}

Figure 2: Closed-loop step responses for Example 1.

TABle 2: Performance indices.

\begin{tabular}{|c|c|c|c|c|c|}
\hline Methods & Loop & $k_{C, i j}$ & $k_{I, i j}$ & $\lambda_{i}$ & $\gamma$ \\
\hline \multirow{3}{*}{ Proposed } & 1 & 0.9939 & 0.2976 & 8.00 & \multirow{3}{*}{0.07} \\
\hline & 2 & -0.1309 & -0.0471 & 12.0 & \\
\hline & 3 & 7.5314 & 0.7602 & 1.00 & \\
\hline \multirow{3}{*}{ SAT [14] } & 1 & 2.7100 & 0.3640 & - & \multirow{3}{*}{0.01} \\
\hline & 2 & -0.3660 & -0.0350 & - & \\
\hline & 3 & 4.5600 & 1.4760 & - & \\
\hline \multirow{3}{*}{ Lee [15] } & 1 & 0.5930 & 0.1730 & - & \multirow{3}{*}{0.06} \\
\hline & 2 & -0.1240 & -0.0430 & - & \\
\hline & 3 & 3.2200 & 0.4210 & - & \\
\hline
\end{tabular}

TABLE 3: Controller parameters.

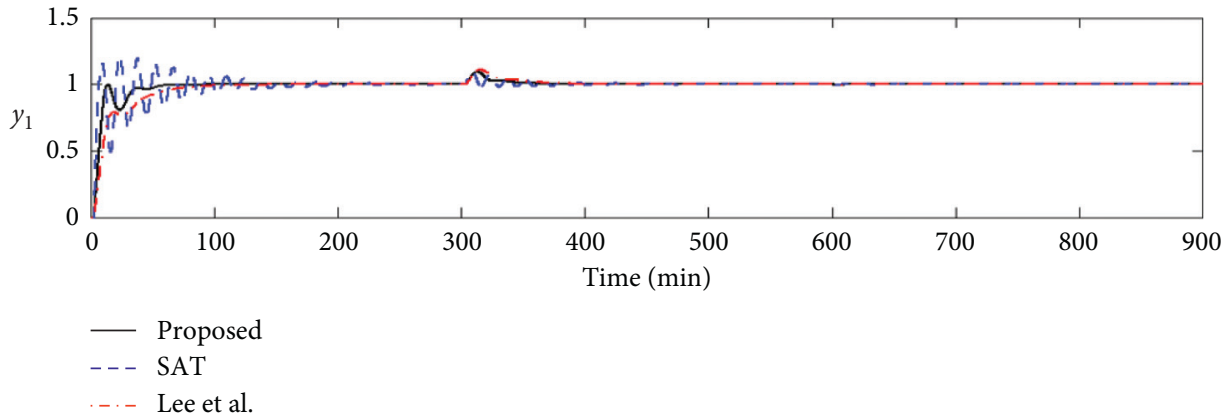

(a)

Figure 3: Continued. 


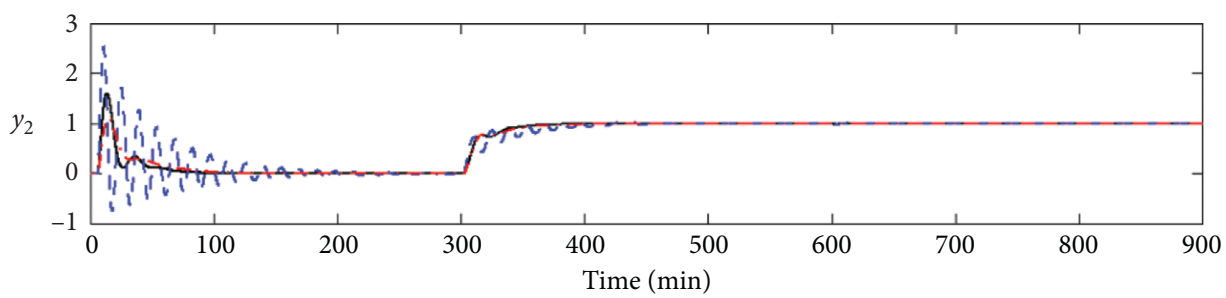

_ Proposed
--- SAT
$-\ldots$ Lee et al.

(b)

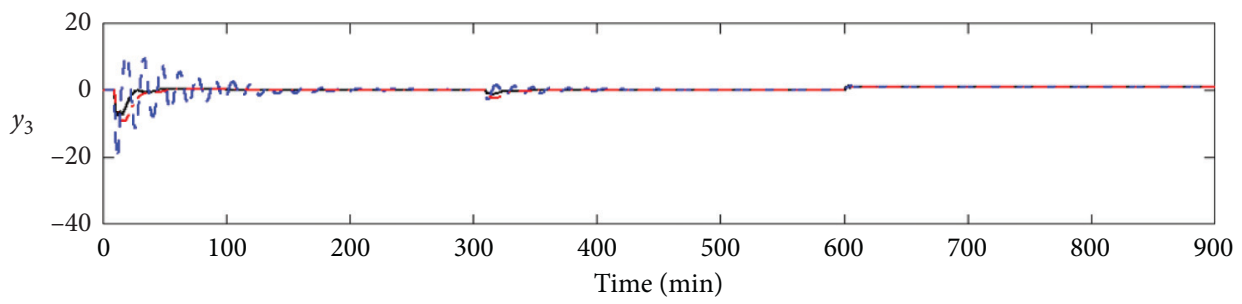

— Proposed

- - - SAT

... Lee et al.

(c)

Figure 3: Closed-loop step responses for Example 2.

TABle 4: Performance indices.

\begin{tabular}{lcccc}
\hline Method & \multicolumn{2}{c}{$\mathrm{IAE}_{s}$} & \multicolumn{2}{c}{ ITAE $_{s}$} \\
& Nominal & $+50 \%$ & Nominal & 3713.8710 \\
Proposed & 180.9597 & 160.7001 & 4431.40 & 46454.8606 \\
SAT & 696.4530 & 2233.640 & 40341.5 & 5722.0498 \\
Lee & 274.4191 & 223.4985 & 7456.57 & \\
\hline
\end{tabular}

Table 5: Controller parameters.

\begin{tabular}{|c|c|c|c|c|c|c|}
\hline Methods & Loop & $k_{C, i j}$ & $k_{I, i j}$ & $k_{D, i j}$ & $\lambda_{i}$ & $\gamma$ \\
\hline \multirow{4}{*}{ Proposed } & 1 & 2.4881 & 0.0915 & - & 7.0 & \multirow{4}{*}{0.15} \\
\hline & 2 & 3.2068 & 0.2241 & - & 2.0 & \\
\hline & 3 & 4.3252 & 0.1664 & - & 1.0 & \\
\hline & 4 & 5.7641 & 0.0340 & - & 5.0 & \\
\hline \multirow{4}{*}{$\mathrm{He}$} & 1 & 3.8840 & 0.0940 & 25.75 & - & \multirow{4}{*}{0.02} \\
\hline & 2 & 2.5490 & 0.0570 & - & - & \\
\hline & 3 & 1.3110 & 0.0710 & - & - & \\
\hline & 4 & 4.2330 & 0.0780 & 23.57 & - & \\
\hline \multirow{4}{*}{ Shen } & 1 & 3.0503 & 0.0739 & 20.23 & - & \multirow{4}{*}{0.03} \\
\hline & 2 & 2.5020 & 0.0561 & - & - & \\
\hline & 3 & 1.5450 & 0.0835 & - & - & \\
\hline & 4 & 1.7530 & 0.0634 & 19.18 & - & \\
\hline
\end{tabular}

performance. This shows that the proposed PI controller yields fast, satisfying and well-balance response, though the model dimension is high.

Table 6 shows the robustness performance of the proposed methods when the process gain is increased by perturbation uncertainty of $+50 \%$. From Table 6 , we can see that the $\mathrm{IAE}_{s}$ and ITAE values obtained by the new method is smaller than the other methods, which further verify that the proposed method provides with good robust performance for high dimension. 


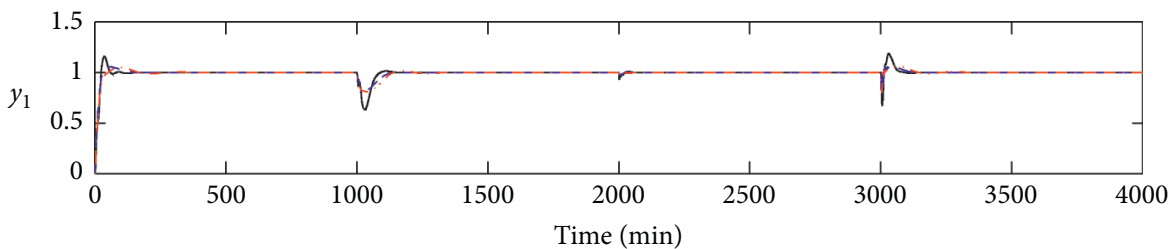

- Proposed

- - - He et al.

...- Shen et al.

(a)

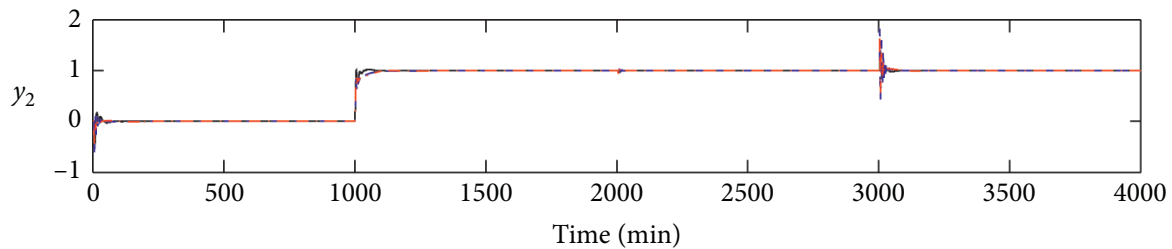

Proposed

- - - He et al.

-.. Shen et al.

(b)

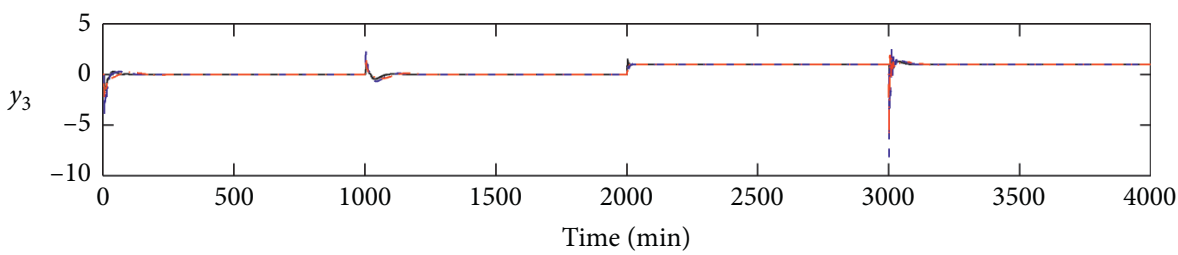

- Proposed

- - - He et al.

...- Shen et al.

(c)

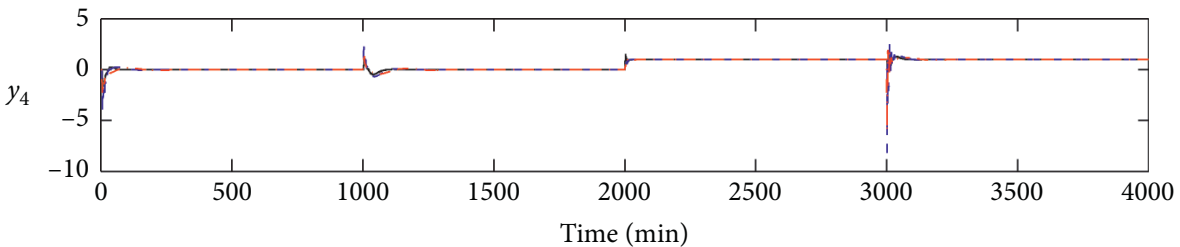

- Proposed

- - - He et al.

.... Shen et al.

(d)

FIgURE 4: Closed-loop step responses for Example 3.

TABle 6: Performance indices.

\begin{tabular}{lcccr}
\hline Methods & \multicolumn{2}{c}{$\mathrm{IAE}_{s}$} & \multicolumn{2}{c}{ ITAE $_{s}$} \\
& Nominal & $+50 \%$ & Nominal & 14245.40 \\
\hline Proposed & 234.4048 & 219.1850 & 16824.34 & 20731.42 \\
He & 278.6150 & 447.8400 & 10393.18 & 22231.41 \\
Shen & 316.1688 & 323.3098 & 25146.40 & \\
\hline
\end{tabular}




\section{Conclusions}

In this paper, an effective controller design method for multivariable systems has been developed. Based on the properties of the ETF and the IMC design principle, the decentralized PI controller is established directly by the proposed method. This method analytically derivates the relationships between controller parameters and the openloop process transfer function. Three different simulation examples have proved the effectiveness of this method and it also can be applied to high-dimensional system. Further work will focus on designing the decentralized controller for nonsquare multivariable systems.

\section{Data Availability}

The data used to support the findings of this study are included within the article.

\section{Conflicts of Interest}

The authors declare that there are no conflicts of interest regarding the publication of this paper.

\section{Acknowledgments}

This work was supported by the Natural Science Foundation of China, under Grant no. 61773183.

\section{References}

[1] S. Gigi and A. K. Tangirala, "Quantification of interaction in multiloop control systems using directed spectral decomposition," Automatica, vol. 49, no. 5, pp. 1174-1183, 2013.

[2] H.-P. Huang, J.-C. Jeng, C.-H. Chiang, and W. Pan, "A direct method for multi-loop PI/PID controller design," Journal of Process Control, vol. 13, no. 8, pp. 769-786, 2003.

[3] Y. Shen, W.-J. Cai, and S. Li, "Multivariable process control: decentralized, decoupling, or sparse?" Industrial \& Engineering Chemistry Research, vol. 49, no. 2, pp. 761-771, 2010.

[4] T. N. L. Vu and M. Lee, "Independent design of multi-loop PI/ PID controllers for interacting multivariable processes," Journal of Process Control, vol. 20, no. 8, pp. 922-933, 2010.

[5] K. H. Estévez-Sánchez, A. Sampieri-Croda, M. A. GarcíaAlvarado, and I. I. Ruiz-López, "Design of multiloop PI controllers based on quadratic optimal approach," ISA Transactions, vol. 70, pp. 338-347, 2017.

[6] S. Cha, D. Chun, and J. Lee, "Two-step IMC-PID method for multiloop control system design," Industrial \& Engineering Chemistry Research, vol. 41, no. 12, pp. 3037-3041, 2002.

[7] T. N. L. Vu and M. Lee, "Multi-loop PI controller design based on the direct synthesis for interacting multi-time delay processes," ISA Transactions, vol. 49, no. 1, pp. 79-86, 2010.

[8] Y. Lee, S. Park, M. Lee, and C. Brosilow, "PID controller tuning for desired closed-loop responses for SI/SO systems," AIChE Journal, vol. 44, no. 1, pp. 106-115, 1998.

[9] Q. Wang, W. J. Cai, and M. J. He, "Equivalent transfer function method for PI/PID controller design of Multivariable processes," Journal of Process Control, vol. 17, no. 18, pp. 665-673, 2007.
[10] Q.-G. Wang, Y. Zhang, and M.-S. Chiu, "Non-interacting control design for multivariable industrial processes," Journal of Process Control, vol. 13, no. 3, pp. 253-265, 2003.

[11] T. Liu, W. Zhang, and F. Gao, "Analytical decoupling control strategy using a unity feedback control structure for MIMO processes with time delays," Journal of Process Control, vol. 17, no. 2, pp. 173-186, 2007.

[12] J. Lee, D. Hyun Kim, and T. F. Edgar, "Static decouplers for control of multivariable processes," AICHE Journal, vol. 51, no. 10, pp. 2712-2720, 2005.

[13] D. Chen and D. E. Seborg, "Design of decentralized PI control systems based on Nyquist stability analysis," Journal of Process Control, vol. 13, no. 1, pp. 27-89, 2003.

[14] A. P. Loh, C. C. Hang, C. K. Quek, and V. U. Vasnani, "Autotuning of multivariable PI controllers using relay feedback," Industrial \& Engineering Chemistry Research, vol. 32, no. 6, pp. 1102-1107, 1993.

[15] M. Lee, K. Lee, C. Kim, and J. Lee, "Analytical design of multiloop PID controllers for desired closed-loop responses," AICHE Journal, vol. 50, no. 7, pp. 1631-1635, 2004.

[16] M.-J. He, W.-J. Cai, and B.-F. Wu, "Design of decentralized IMC-PID controller based on dRI analysis," AICHE Journal, vol. 52, no. 11, pp. 3852-3863, 2006.

[17] S. Skogestad, "Simple analytic rules for model reduction and PID controller tuning," Journal of Process Control, vol. 13, no. 4, pp. 291-309, 2003. 